\title{
Sex-Specific Analysis at Two Time Points in Three High-Impact Orthopaedic Sports Medicine Journals
}

\author{
Arianna L. Gianakos, D.O., Patrick Szukics, D.O., Nicole George, D.O., \\ Sherif Elkattawy, D.O., Dawn M. LaPorte, M.D., and Mary K. Mulcahey, M.D.
}

\begin{abstract}
Purpose: To determine how well the orthopaedic sports medicine literature reported sex-specific analysis (SSA) in 2011 and 2016. Methods: The 3 highest-impact orthopaedic sports medicine subspecialty journals (American Journal of Sports Medicine; Arthroscopy; and Knee Surgery, Sports Traumatology, Arthroscopy) were selected for review. Two independent investigators reviewed all journal issues published during 2 different calendar years (2011 and 2016). All randomized controlled, prospective and retrospective group, and case-control studies were included. Studies were stratified into those that involved SSA, where sex was a variable in a multifactorial statistical model, and those that only reported sex as a demographic characteristic or used sex-matched groups without further analysis. Results: A total of 960 studies evaluating 3,400,569 patients met criteria and were included in this review. Although $44.4 \%$ of the overall study population was female, only 293 $(30.5 \%)$ studies included patient sex as variable in a multifactorial statistical model. The proportion of studies that performed SSA did not differ between 2011 (29.6\%) and 2016 (31.1\%; P=.607), although publications from American Journal of Sports Medicine were likely to report SSA $(P<.05)$. Of the 293 studies that reported SSA, $91(31 \%)$ demonstrated a significant difference in outcomes. The most commonly reported differences were in outcomes following anterior cruciate ligament, medial patellofemoral ligament, and posterior cruciate ligament reconstruction; autograft preparation; postoperative use of opiates following arthroscopy; and recovery after sports-related concussions. Conclusions: Although most sports medicine studies include approximately 50\% female and 50\% male patients, statistical analysis differentiating the 2 subsets is not routinely performed. Only $30.5 \%$ of all studies performed SSA, $31 \%$ of which reported a statistically significant difference in the data when comparing outcomes between male and female patients with the same treatment modalities. Clinical Relevance: The current study demonstrates that the orthopaedic sports medicine subspecialty literature is lacking in reporting SSA, and that there has been minimal improvement over a 5-year time period. In addition, this study highlights the high percentage of significant findings within the studies that performed SSA and underscores the differences in sex-specific injury patterns and treatment outcomes.
\end{abstract}

$\mathbf{T}$ he increase in female participation in competitive sports has been accompanied by a rise in the number of sports-related injuries in this population. ${ }^{1}$ Female athletes have become more involved in what were initially deemed "male"-dominant sports with greater physical demands (basketball, hockey, boxing, weight lifting, martial arts, and football), and therefore

From the Department of Orthopaedic Surgery, Robert Wood Johnson Barnabas Health-Jersey City Medical Center, Jersey City (A.L.G., S.E.); and Department of Orthopaedic Surgery, Rowan School of Osteopathic Medicine, Stratford (P.S.), New Jersey; Department of Orthopaedic Surgery, Aultman Hospital, Canton, Ohio (N.G.); Department of Orthopaedic Surgery, Johns Hopkins University School of Medicine, Baltimore, Maryland (D.L.); and Department of Orthopaedic Surgery, Tulane University School of Medicine, New Orleans, Louisiana (M.K.M.), U.S.A.

The authors report the following potential conflicts of interest or sources of funding: M.K.M. reports board or committee member: American Academy of Orthopaedic Surgeons, American Orthopaedics Society for Sports Medicine, Arthroscopy Association of North America, and Ruth Jackson Orthopaedic the data evaluating female-specific outcomes within these sports are lacking.

Previous literature has identified differences in sex-related risk factors contributing to injuries including anterior cruciate ligament (ACL) tear, stress fracture, multidirectional shoulder instability, and concussion. ${ }^{1,2}$ In addition, several studies have

Society; and paid speaker or presented for Arthrex, outside the submitted work; Full ICMJE author disclosure forms are available for this article online, as supplementary material.

Received July 18, 2019; accepted February 7, 2020.

Address correspondence to Arianna L. Gianakos, D.O., 414 Grand St., Suite 14, Jersey City, NJ 07302.E-mail: algianakos@gmail.com

(C) 2020 THE AUTHORS. Published by Elsevier Inc. on behalf of the Arthroscopy Association of North America. This is an open access article under the CC BY-NC-ND license (http://creativecommons.org/licenses/by-nc-nd/4.0/). 2666-061X/19882

https://doi.org/10.1016/j.asmr.2020.02.002 
demonstrated an increase in overuse injuries in female athletes when compared with their male counterparts. $^{3-5}$ Inherent physiologic differences exist between male and female athletes. Remvig et al. ${ }^{6}$ reported an increase in hypermobility in female patients compared with male patients. Several authors have also shown an influence of sex hormone levels on collagen turnover, muscle tissue turnover, and ligament and tendon injury risk. ${ }^{7,8}$ It has been well established that any prolonged reduction in estrogen levels can lead to increased degradative activity of bone resulting in a reduced bone mineral density, leading to osteoporosis and an increased susceptibility to fractures. ${ }^{2}$ Similarly, young female athletes may suffer from injuries due to hormonal deficits accompanying the female athletic triad. Lastly, psychological factors and response to pain also can impact the effect that an injury has on an athlete, and differences between male and female responses are poorly understood., ${ }^{2,9}$

Although recent literature has attempted to identify differences in the prevalence of sports injuries between male and female athletes, few studies have evaluated sex-specific injury patterns and treatment outcomes. $^{1,2,7,8}$ Proper identification of the disparities between male and female athletes is critical to better individualize treatment and prevention strategies. The purposes of this study were to determine how well the sports medicine orthopaedic literature reported sex-specific analysis (SSA) in 2011 and 2016, and whether there was an increasing trend in reporting SSA. We hypothesized that SSA in the current orthopaedic sports medicine literature would be underreported and that there would be a limited increase in the reporting of sex-related differences over the last 5 years.

\section{Methods}

\section{Article Selection}

The 3 highest-impact orthopaedic sports medicine subspecialty journals (American Journal of Sports Medicine [AJSM]; Arthroscopy; and Knee Surgery, Sports, Traumatology, Arthroscopy [KSSTA]) were chosen for review. Journal issues published during all months in 2011 and 2016 were assessed for the use of SSA by 2 independent reviewers. All randomized controlled trials, prospective or retrospective group studies, and case-control studies that evaluated both male and female populations were considered for study inclusion. Case reports and case series, review articles, biomechanical studies, and animal studies were excluded. Other inclusion criteria included the availability of the full-text manuscript. All articles were then assessed to determine whether SSA was performed. For the purposes of the present study, SSA was defined as the use of sex as a variable in a multifactorial statistical model. Studies were selected and independently assessed by 2 orthopaedic surgery residents (postgraduate years 2 and 4). Differences between the reviewers were discussed until agreement was achieved and the senior author was consulted in the event of persistent disagreement. Studies that did not consider women for the study population were not included in this analysis.

\section{Data Collection}

Data was then collected and recorded on all journal articles that met the inclusion and exclusion criteria. Variables collected included the total number of patients, total number of female patients, specific body part evaluated, injury, surgical intervention, type of sex reporting, and results of the SSA (only if applicable).

\section{Statistical Analysis}

The studies were then stratified into 2 groups: (1) those that reported SSA, and (2) those that either solely reported sex as a demographic characteristic or that used sex-matched groups without further analysis. The primary outcome of interest was the use of SSA, as investigated over time, between different subspecialty journals, and between different body parts. $\chi^{2}$ analysis was used to evaluate categorical variables, and Student $t$ testing was used to compare continuous variables. Statistical analysis was then performed comparing these 2 groups. All statistical analysis was performed using SPSS, version 25 (IBM Corp., Armonk, NY). A P value of less than .05 was designated as the threshold for statistical significance.

\section{Results}

A total of 2001 studies were evaluated by the 2 independent reviewers, including 671 from AJSM, 489 from Arthroscopy, and 841 from KSSTA. We excluded a total of 1041 total studies, including 302 from AJSM, 292 from Arthroscopy, and 447 from KSSTA that did not meet criteria. A total of 960 studies evaluating $3,400,569$ patients met criteria and were included in this review (Table 1). The total study population was comprised of $44.4 \%$ female patients, with similar proportions reported in each of the 3 journals. Overall, 293 studies $(30.5 \%)$ included sex as variable in a multifactorial statistical model: $131(35.5 \%)$ of the included AJSM publications, $57(28.9 \%)$ of the publications from Arthroscopy, and $105(26.6 \%)$ of the studies from KSSTA. There was no significant change in SSA reporting from 2011 (113/382, 29.6\%) to $2016(180 / 578,31.1 \% ; P=.607)$ (Table 2).

However, AJSM consistently reported SSA at greater rates than the other 2 journals. Specifically, AJSM was more likely and KSSTA was less likely to include SSA than Arthroscopy $(35.5 \%$ and $26.6 \%$, respectively, vs $28.9 \% ; P=.026$ ) during both years combined, and KSSTA was less likely than AJSM and Arthroscopy to 
Table 1. Descriptive Analysis of Orthopaedic Sports Medicine Journal Articles Published In 2011 and 2016 Used for Evaluation of Sex-Specific Analysis

\begin{tabular}{|c|c|c|c|c|}
\hline & AJSM & Arthroscopy & KSSTA & Total \\
\hline Total number of studies analyzed & 369 & 197 & 394 & 960 \\
\hline $\begin{array}{l}\text { Number of studies in which sex was included as a } \\
\text { variable in a multifactorial statistical model }(\%)\end{array}$ & $131(35.5 \%)$ & $57(28.9 \%)$ & $105(26.6 \%)$ & $293(30.5 \%)$ \\
\hline $\begin{array}{l}\text { Number of studies in which sex was only included as a } \\
\text { demographic variable }(\%)\end{array}$ & $220(59.6 \%)$ & $129(65.5 \%)$ & $274(69.5 \%)$ & $623(64.9 \%)$ \\
\hline $\begin{array}{l}\text { Number of studies involving sex-adjusted analysis or } \\
\text { sex-matched groups, but no specific analysis (\%) }\end{array}$ & $18(4.9 \%)$ & $11(5.6 \%)$ & $15(3.8 \%)$ & $44(4.6 \%)$ \\
\hline Total number of patients analyzed & $\begin{array}{c}2,563,952(75.4 \% \text { of } \\
\text { overall total })\end{array}$ & $\begin{array}{l}750,703(22.1 \% \text { of } \\
\text { overall total })\end{array}$ & $\begin{array}{c}85,914(2.5 \% \text { of } \\
\text { overall total })\end{array}$ & $3,400,569$ \\
\hline Total number of female patients analyzed ( \%) & $\begin{array}{c}1,109,278(43.3 \% \text { of } \\
\text { AJSM total })\end{array}$ & $\begin{array}{l}364,055(48.5 \% \text { of } \\
\text { Arthroscopy total })\end{array}$ & $\begin{array}{l}37,369(43.5 \% \text { of } \\
\text { KSSTA total })\end{array}$ & $\begin{array}{l}1,510,702(44.4 \% \text { of } \\
\text { overall total })\end{array}$ \\
\hline
\end{tabular}

AJSM, American Journal of Sports Medicine; KSSTA, Knee Surgery, Sports, Traumatology, Arthroscopy.

include SSA in $2016(25.9 \%$ vs 36.1 and $33.9 \%$, respectively; $P=.048$ ) (Table 3 ; Fig 1 ).

Of the 293 studies that reported SSA, 90 (31\%) demonstrated a significant difference in outcomes between male and female patients. The majority of articles that demonstrated a significant difference were evaluating the knee (58 articles, 64\%) (Table 4). Differences in outcome also were found in studies of the hip (15 articles, $16 \%$ ), shoulder ( 15 articles, $16 \%$ ), and foot and ankle ( 2 articles, $2 \%$ ) (Table 4 ). Overall, ACL injury (29/58 studies, i.e., $50 \%$ of all significant knee studies) was the most common subtopic in which a significant difference was identified after SSA. The hip and shoulder categories in which such differences were most common were femoroacetabular impingement $(8 / 15$ studies, $53 \%$ of all significant studies) and the rotator cuff $(9 / 15$ studies, or $60 \%$ of all significant studies), respectively.

\section{Discussion}

This study demonstrates that the current orthopaedic sports medicine subspecialty literature is lacking in reporting SSA. There has been minimal improvement when examining 2 time points that were 5 years apart. In addition, this study highlights that $30.5 \%$ of studies that performed SSA reported significant differences. This finding underscores the differences in sex-specific injury patterns and treatment outcomes and highlights the need for more research that includes SSA.

The increasing participation in female sports over the last several decades poses a challenge for orthopaedic surgeons, as female athletes may be at greater risk than their male counterparts for sustaining certain injuries. Patterns of injury are generally assumed to be sportspecific rather than sex-specific. Although overuse injuries are more common in female athletes when compared with male athletes, there has been limited literature establishing prevention protocols for young female athletes. ${ }^{3,5}$ An example includes the biomechanics of a windmill pitch in softball when compared with the overhead pitch in baseball. The literature primarily has focused on injuries related to the overhead throw pattern, with little evidence based outcomes evaluating the mechanics of the windmill throw in female athletes. ${ }^{10}$ These types of discrepancies underscore the importance of more focused sex-related research within the athletic population. In addition, men have traditionally participated in the majority of sports, and consequently as a result, data evaluating training, equipment, and both nonoperative and operative interventions have been largely biased based on male experience.

The included articles within this study highlight the differences in the management and treatment outcomes in male and female patients. It has been well-established within epidemiologic studies that there is a significantly increased risk of noncontact ACL injury in female athletes when compared with male athletes. ${ }^{1,2,10}$ Intrinsic risk factors including alignment, hyperextension, physiologic rotatory laxity, ACL size, notch size, and hormonal influences have all been attributed as factors contributing to ACL injuries. ${ }^{11}$ Several articles within this study support these known discrepancies underscoring the differences in anatomy. ${ }^{12,13}$ Although equally favorable results have been reported following ACL reconstruction in both male and female patients, studies have demonstrated that female athletes may be less likely to return to competitive play. ${ }^{14}$ Paterno et al. ${ }^{15}$ reported that female patients are 4 times more likely to experience graft retears and 6 times as likely to suffer a subsequent

Table 2. Comparison of Sex-Specific Analysis Between Orthopaedic Sports Medicine Journal Publications in 2011 and 2016

\begin{tabular}{cccc}
\hline & 2011 & 2016 & $P / \chi^{2}$ \\
\hline $\begin{array}{c}\text { Sex-specific analysis } \\
\text { NOT performed }\end{array}$ & $269(70.4 \%)$ & $398(68.9 \%)$ & \\
$\begin{array}{c}\text { Sex-specific analysis } \\
\text { performed }\end{array}$ & $113(29.6 \%)$ & $180(31.1 \%)$ & $.607 / 0.694$ \\
\hline
\end{tabular}


Table 3. Comparison of Sex-Specific Analysis Between Orthopaedic Sports Medicine Journal Publications, Based on Year of Publication

\begin{tabular}{|c|c|c|c|c|}
\hline & AJSM & Arthroscopy & KSSTA & $\mathrm{P} / \chi^{2}$ \\
\hline \multicolumn{5}{|c|}{$\begin{array}{l}\text { Comparison of SSA between different orthopaedic } \\
\text { sports medicine journals in } 2011 \text { and } 2016\end{array}$} \\
\hline Sex-specific analysis NOT performed & $238(64.5 \%)$ & $140(71.1 \%)$ & $289(73.4 \%)$ & \\
\hline Adjusted standardized residuals & \pm 2.6 & \pm 0.5 & \pm 2.2 & $.026 / 7.335$ \\
\hline \multicolumn{5}{|c|}{$\begin{array}{l}\text { Comparison of SSA between different orthopaedic } \\
\text { sports medicine journals in } 2011\end{array}$} \\
\hline \multicolumn{5}{|c|}{$\begin{array}{l}\text { Comparison of SSA between different orthopaedic } \\
\text { sports medicine journals in } 2016 .\end{array}$} \\
\hline Sex-specific analysis NOT performed & $129(63.9 \%)$ & $80(66.1 \%)$ & $189(74.1 \%)$ & \\
\hline Sex-specific analysis performed & $73(36.1 \%)$ & $41(33.9 \%)$ & $66(25.9 \%)$ & \\
\hline Adjusted standardized residuals ${ }^{*}$ & \pm 1.9 & \pm 0.7 & \pm 2.4 & $.048 / 6.066$ \\
\hline
\end{tabular}

NOTE. Boldface indicates statistical significance.

AJSM, American Journal of Sports Medicine; KSSTA, Knee Surgery, Sports, Traumatology, Arthroscopy.

*Values outside of the $95 \%$ confidence interval (adjusted standardized residual \pm 1.96 ) indicate a statistically significant difference between groups.

contralateral injury. Therefore, a better understanding of the factors that play a role in the rehabilitation of these athletes is important to better prepare an athlete to return to competitive play.

Injury patterns and outcome studies are beginning to focus on other ligamentous injuries within the knee, including the posterior cruciate ligament (PCL) and medial patellofemoral ligament (MPFL). Jung et al. ${ }^{16}$ performed a prospective database study evaluating outcomes of 234 male and female patients following PCL reconstructions. The authors reported a significantly greater reduction of posterior tibial displacement after PCL reconstruction in female patients when compared with male patients. Nagano et al. ${ }^{17}$ conducted a knee kinematic and electromyography study evaluating landing mechanics in 18 male athletes and 19 female athletes. The authors reported a significantly larger internal tibial rotation during landing as well as a slower time of maximum vertical ground reaction force after time of foot contact in female athletes when compared with male athletes. Female athletes typically have greater quadriceps activation and lower hamstring contraction, which can result in significant anterior tibial displacement and internal tibial rotation, thereby increasing the risk of ACL rupture. Previous authors suggest that reduced hamstring contraction in female athletes may in fact protect the PCL and ultimately may lead to better postoperative outcomes following PCL reconstruction. ${ }^{16}$

Female sex is an established risk factor for patella instability often leading to patellar dislocation and MPFL injury. ${ }^{18}$ There are numerous techniques for imbrication, repair, and reconstruction of the MPFL, but outcomes remain controversial. Sanchis-Alfonso et al. ${ }^{19}$ performed a study evaluating radiographic techniques to ensure precise anatomic location of the femoral fixation site in MPFL reconstruction. They calculated percentage of anatomic tunnel covered by the tunnel created as previously described by Schöttle et al. ${ }^{20}$ Sixty percent of the male population had greater than $50 \%$ coverage compared with only $20 \%$ of the female population $(P<.001)$, indicating that this method would not be accurate if used when reconstructing the MPFL in female patients. ${ }^{19}$

Although most studies evaluated sex-related differences have focused on knee injuries, there has been increased attention toward discrepancies found within the hip. Sex-based differences in the anatomic features of the hip have been previously described. Studies have demonstrated that women have increased acetabular

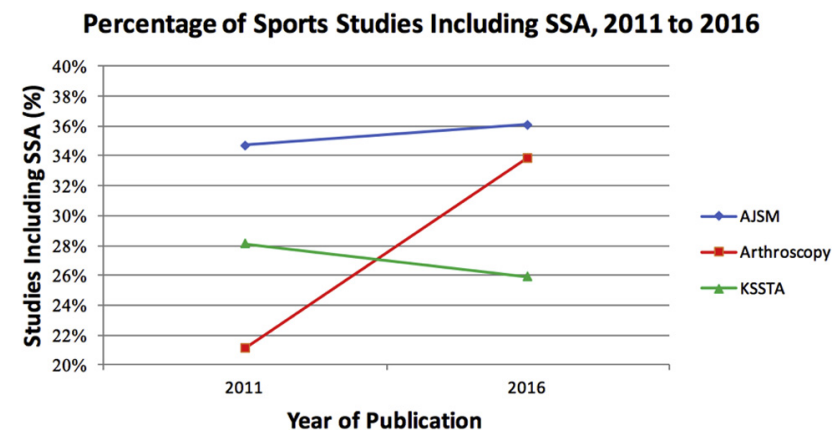

Fig 1. Proportion of orthopaedic sports medicine journal publications from 2011 and 2016 that included SSA, compared by journal. AJSM consistently reported SSA at greater rates than the other 2 journals. Specifically, AJSM was more likely and KSSTA was less likely to include SSA than Arthroscopy $(35.5 \%$ and $26.6 \%$, respectively, vs $28.9 \%$; $P=.026)$ during both years combined, and KSSTA was less likely than AJSM and Arthroscopy to include SSA in 2016 (25.9\% vs 36.1 and $33.9 \%$, respectively; $P=.048)$. (AJSM, American Journal of Sports Medicine; KSSTA, Knee Surgery, Sports, Traumatology, Arthroscopy; SSA, sex-specific analysis.) 
Table 4. Specific Topics Investigated in Orthopaedic Sports Medicine Journal Publications (2011-2016) Evaluated for Sex-Specific Analysis

\begin{tabular}{lr}
\hline Knee $(\mathrm{n}=58)$ & 29 \\
Anterior cruciate ligament & 8 \\
Anatomy & 7 \\
Total knee arthroplasty & 6 \\
Miscellaneous & 4 \\
Cartilage & 4 \\
Meniscus & 2 \\
Patellofemoral joint & 8 \\
Hip (n = 15) & 4 \\
Femoroacetabular impingement & 3 \\
Miscellaneous & \\
Anatomy & 9 \\
Shoulder (n $=15)$ & 4 \\
Rotator cuff & 3 \\
Instability & 1 \\
Biceps tendon & 1 \\
Foot and ankle $(\mathrm{n}=2)$ & \\
Achilles tendon & \\
Foot orthosis &
\end{tabular}

version, increased femoral anteversion, and decreased lateral center edge angle, all of which create differences in the progression of hip pathology when compared to their male counterparts. ${ }^{21}$ Several studies have demonstrated an increased incidence of the development of femoroacetabular impingement in male patients and patients with larger alpha angles resulting in a significantly greater risk of grade IV cartilaginous defects. $^{22}$ Male sex also has been significantly associated with labral tears and acetabular chondromalacia and recently has been associated with a greater progression of disease severity. ${ }^{23}$ Therefore, knowledge of the differences in the underlying hip pathology and progression of the disease can influence treatment strategies for male and female patients.

Although the orthopaedic literature has demonstrated significant differences in male and female anatomy and injuries, data evaluating the progression of pathology as well as the outcomes of various treatment strategies are lacking. A previous study by Hettrich et al. ${ }^{24}$ demonstrated improvement in the reporting of SSA in the general orthopaedic literature from 2000 to 2010 but concluded that $70 \%$ of the clinical studies included failed to perform proper statistical analysis based on sex. Sugimoto et al. ${ }^{25}$ recently published a study in Lancet demonstrating that $67 \%$ of clinical medicine studies, $69 \%$ of public health research studies, and only $31 \%$ of biomedical research studies performed sex-related reporting in 2016. The authors also found that female first or last authors had a greater probability of sex-related reporting than male authors, and journals with high impact factors were less likely to report sex. It is critical to not only include both male and female sex participants in studies, but it is important to evaluate for differences to limit biases in the management and treatment of different diseases and pathologies.

\section{Limitations}

There are several limitations to this study. First, data were only reported for the years 2011 and 2016. Different trends may have been observed if all issues between 2011 and 2016 were included, although there is a precedent for performing studies evaluating differences between 2 years. However, we believe this is an accurate representation of the trends. Another limitation is that this study only included 3 sports medicine journals: AJSM, KSSTA, and Arthroscopy. Inclusion of a greater number of journals would increase the number of articles evaluated. There is also a potential for selection bias, as we did not analyze other high-impact journals in the general orthopaedic literature and screen them for sports medicine-related publications. In addition, this study only evaluated outcomes within the sports medicine subspecialty literature, therefore including all areas of orthopaedic surgery would result in a more generalized analysis across all subspecialties. Lastly, another limitation is the lack of knowledge of which studies and/or what percentage of studies should include a SSA, as this is not practical for all studies. For certain studies and certain conditions, it may not be practical to obtain sufficient patient numbers to perform a multivariable or SSA.

\section{Conclusions}

Although most sports medicine studies include approximately $50 \%$ female and $50 \%$ male patients, statistical analysis differentiating the 2 subsets is not routinely performed. Only $30.5 \%$ of all studies performed SSA, $31 \%$ of which reported a statistically significant difference in the data when comparing outcomes between male and female patients with the same treatment modalities.

\section{References}

1. Carter CW, Ireland ML, Johnson AE, et al. Sex-based differences in common sports injuries. J Am Acad Orthop Surg 2018;26:447-454.

2. Goldring AE, Ashok AP, Casey EK, Mulcahey MK. Key components and potential benefits of a comprehensive approach to women's musculoskeletal health. Phys Sportsmed 2016;44:417-424.

3. Schroeder AN, Comstock RD, Collins CL, Everhart J, Flanigan D, Best TM. Epidemiology of overuse injuries among high-school athletes in the United States. J Pediatr 2015;166:600-606.

4. Vincent HK, Zdziarski LA, Vincent KR. Review of lacrosserelated musculoskeletal injuries in high school and collegiate players. Sports Health 2015;7:448-451.

5. Yang J, Tibbetts AS, Covassin T, Cheng G, Nayar S, Heiden E. Epidemiology of overuse and acute injuries among competitive collegiate athletes. J Athl Train 2012;47:198-204.

6. Remvig L, Jensen DV, Ward RC. Epidemiology of general joint hypermobility and basis for the proposed criteria for 
benign joint hypermobility syndrome: Review of the literature. J Rheumatol 2007;34:804-809.

7. Frank RM, Romeo AA, Bush-Joseph CA, Bach BR Jr. Injuries to the female athlete in 2017: Part I: General considerations, concussions, stress fractures, and the female athlete triad. JBJS Rev 2017;5(10):e4.

8. Hansen M, Kjaer M. Sex hormones and tendon. Adv Exp Med Biol 2016;920:139-149.

9. Templeton KJ, Hame SL, Hannafin JA, Griffin LY, Tosi LL, Shields NN. Sports injuries in women: Sex- and genderbased differences in etiology and prevention. Instr Course Lect 2008;57:539-552.

10. Frank RM, Romeo AA, Bush-Joseph CA, Bach BR Jr. Injuries to the Female athlete in 2017: Part II: Upper and lower-extremity injuries. JBJS Rev 2017;5:e5.

11. Ireland ML. The female ACL: why is it more prone to injury? Orthop Clin North Am 2002;33:637-651.

12. Erkocak OF, Kucukdurmaz F, Sayar S, Erdil ME, Ceylan HH, Tuncay I. Anthropometric measurements of tibial plateau and correlation with the current tibial implants. Knee Surg Sports Traumatol Arthrosc 2016;24:2990-2997.

13. Lim H-C, Lee Y-G, Wang J-H, et al. Anterior and posterior knee laxity in a young adult Korean population. Knee Surg Sports Traumatol Arthrosc 2011;19:1890-1894.

14. Brophy RH, Schmitz L, Wright RW, et al. Return to play and future ACL injury risk after ACL reconstruction in soccer athletes from the Multicenter Orthopaedic Outcomes Network (MOON) group. Am J Sports Med 2012;40: 2517-2522.

15. Paterno MV, Rauh MJ, Schmitt LC, Ford KR, Hewett TE. Incidence of contralateral and ipsilateral anterior cruciate ligament (ACL) injury after primary ACL reconstruction and return to sport. Clin J Sport Med 2012;22:116-121.

16. Jung TM, Lubowicki A, Wienand A, Wagner M, Weiler A. Knee stability after posterior cruciate ligament reconstruction in female versus male patients: A prospective matched-group analysis. Arthroscopy 201 1;27:399-403.
17. Nagano Y, Ida H, Akai M, Fukubayashi T. Gender differences in knee kinematics and muscle activity during single limb drop landing. Knee 2007;14:218-223.

18. Stein BES, Shubin Stein BE, Gruber S, Brady JM. MPFL in first-time dislocators. Curr Rev Musculoskelet Med 2018;11: 182- 187.

19. Sanchis-Alfonso V, Ramirez-Fuentes C, MontesinosBerry E, Aparisi-Rodriguez F, Martí-Bonmatí L. Does radiographic location ensure precise anatomic location of the femoral fixation site in medial patellofemoral ligament surgery? Knee Surg Sports Traumatol Arthrosc 2016;24: 2838-2844.

20. Schöttle PB, Schmeling A, Rosenstiel N, Weiler A. Radiographic landmarks for femoral tunnel placement in medial patellofemoral ligament reconstruction. Am J Sports Med 2007;35:801-804.

21. Frank RM, Lee S, Bush-Joseph CA, Kelly BT, Salata MJ, Nho SJ. Improved outcomes after hip arthroscopic surgery in patients undergoing T-capsulotomy with complete repair versus partial repair for femoroacetabular impingement: A comparative matched-pair analysis. Am J Sports Med 2014;42:2634-2642.

22. Bhatia S, Nowak DD, Briggs KK, Patterson DC, Philippon MJ. Outerbridge Grade IV Cartilage lesions in the hip identified at arthroscopy. Arthroscopy 2016;32: 814-819.

23. Nepple JJ, Carlisle JC, Nunley RM, Clohisy JC. Clinical and radiographic predictors of intra-articular hip disease in arthroscopy. Am J Sports Med 2011;39:296-303.

24. Hettrich CM, Hammoud S, LaMont LE, Arendt EA, Hannafin JA. Sex-specific analysis of data in high-impact orthopaedic journals: how are we doing? Clin Orthop Relat Res 2015;473:3700-3704.

25. Sugimoto CR, Ahn Y-Y, Smith E, Macaluso B, Larivière V. Factors affecting sex-related reporting in medical research: A cross-disciplinary bibliometric analysis. Lancet 2019;393:550-559. 